\title{
Primary Epiploic Appendagitis: Solving the Puzzle of a Benign Acute Abdomen
}

\author{
Neeraj Nagaich ${ }^{1,4 *}$, Radha Sharma ${ }^{2}$, Anshuman Kaushal ${ }^{3}$ and Subhash Nepalia ${ }^{4}$ \\ ${ }^{1}$ Department of Gastroenterology Fortis Escorts Hospital, India \\ ${ }^{2}$ Department of Pathology RUHS College of medical sciences, India \\ ${ }^{3}$ Department of Artemis Hospital Gurgaon, India \\ ${ }^{4}$ Department of Gastroenterology SMS Medical College, India
}

Received: January 24, 2018; Published: February 15, 2018

*Corresponding author: Neeraj Nagaich, Department of Gastroenterology Fortis Escorts Hospital, India, Tel: 919414600141; Email: drneerajn@gmail.com

\begin{abstract}
Background: Acute Epiploic Appendagitis is a self limiting disease due to ischemic infarction of an epiploic appendix. Diagnosis is difficult clinically due to the lack of pathognomonic clinical features, a sudden onset of sharp localized pain either in the left or right iliac fossa with minimal gastrointestinal symptoms, which can simulate a surgical clinical picture. Awareness of imaging findings of this entity is important to arrive at a correct diagnosis and to avoid unnecessary hospitalization and surgery.
\end{abstract}

Methods: 25 patients diagnosed with EA were evaluated and analyzed for demographic factors clinical presentation and diagnostic radiological features. Comparison was also done with data in patients of earlier reported series.

Results: 25 patients ( 7 females and 18 males, average age: 44.6 years, range: $23-74$ years) were diagnosed with symptomatic EA. Abdominal pain was the leading symptom, the pain being localized in the left (13 patients, $54 \%$ ) and right (5 patients, 19\%) lower quadrant. Except two all patients were a febrile, and with the exception of three patients, nausea, vomiting, and diarrhea were not present. CRP was slightly increased (mean: $1.6 \mathrm{mg} / \mathrm{DL}$ ) in seven patients (35\%). Computed tomography findings specific for EA were present in five patients. Gastrointestinal symptoms such as nausea and vomiting were infrequent, and localized tenderness without peritoneal irritation was common. In all cases except two, a pericolic fatty mass with a hyper attenuated ring was observed on computed tomography. four patient (14\%) with left PEA showed leukocytosis,

Conclusion: Epiploic appendagitis was more frequent in males. Abdominal pain was located in the lower quadrant, more predominant in the left than right. Blood tests were normal except for increased levels of C-reactive protein. Diagnosis was made mostly preoperatively due to imaging tests, avoiding unnecessary surgical intervention. In patients with localized, sharp, acute abdominal pain not associated with other symptoms such as nausea, vomiting, fever or atypical laboratory values, the diagnosis of EA should be considered. In patients with acute abdomen \& localized tenderness without associated symptoms or leukocytosis, a high index of suspicion for PEA is necessary. Preoperative diagnosis may avoid unnecessary surgery.

\section{Introduction}

Epiploic appendages are visceral peritoneal out-pouching of colon containing fat and blood vessels Epiploic appendages are pedunculated, leaf-like fat structures wrapped by serosa. The colon has approximately 50 to 100 and $2-5 \mathrm{~cm}$ long epiploic appendages. They protrude from the anterior and posterior portions of the large intestine along the tenia, libera. Although their role is not precisely understood, they are believed to play a protective role as cushions, localized prevention against infection and as energy reservoirs during starvation [1].

Epiploic appendages are nourished by small arteries and veins. Since they are pedunculated and rich in mobility, they are prone to ischemic infarction due to torsion. This is the reported pathomechanism of epiploic appendagitis [2]. However, secondary epiploic appendagitis is caused by the inflammation of adjacent organs, such as the colon diverticulum, gallbladder, and appendix [3]. Epiploic appendages exist along the entire length of the colon. Therefore, it is necessary to differentiate epiploic appendagitis from many other diseases. Diverticulitis, appendicitis, and omental infarction are often difficult to differentiate from epiploic appendagitis. Other potential diagnoses include tuberculous peritonitis, neoplasm, urachal cyst, mesenteric panniculitis, and trauma. Appendagitis is a benign and self-limiting condition of epiploic appendages [4]. 

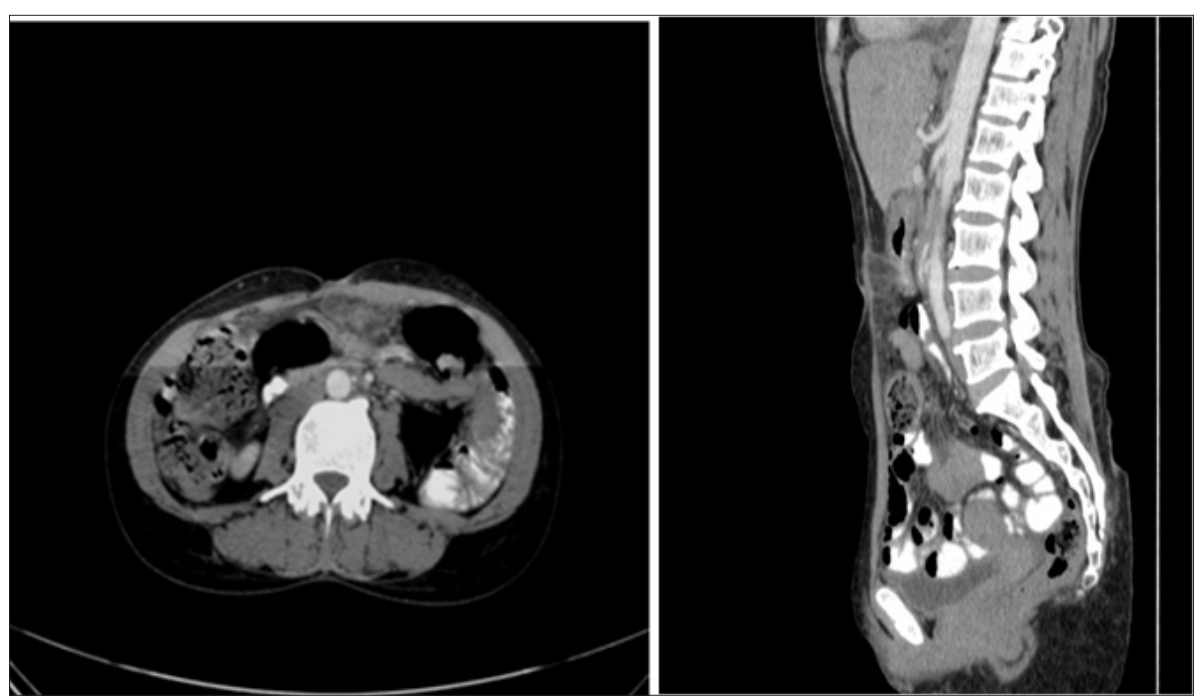

Figure 1: 1A \&1B Focal fat stranding with inflammatory changes in the left para-sagittal and paraumbilical, omental region.

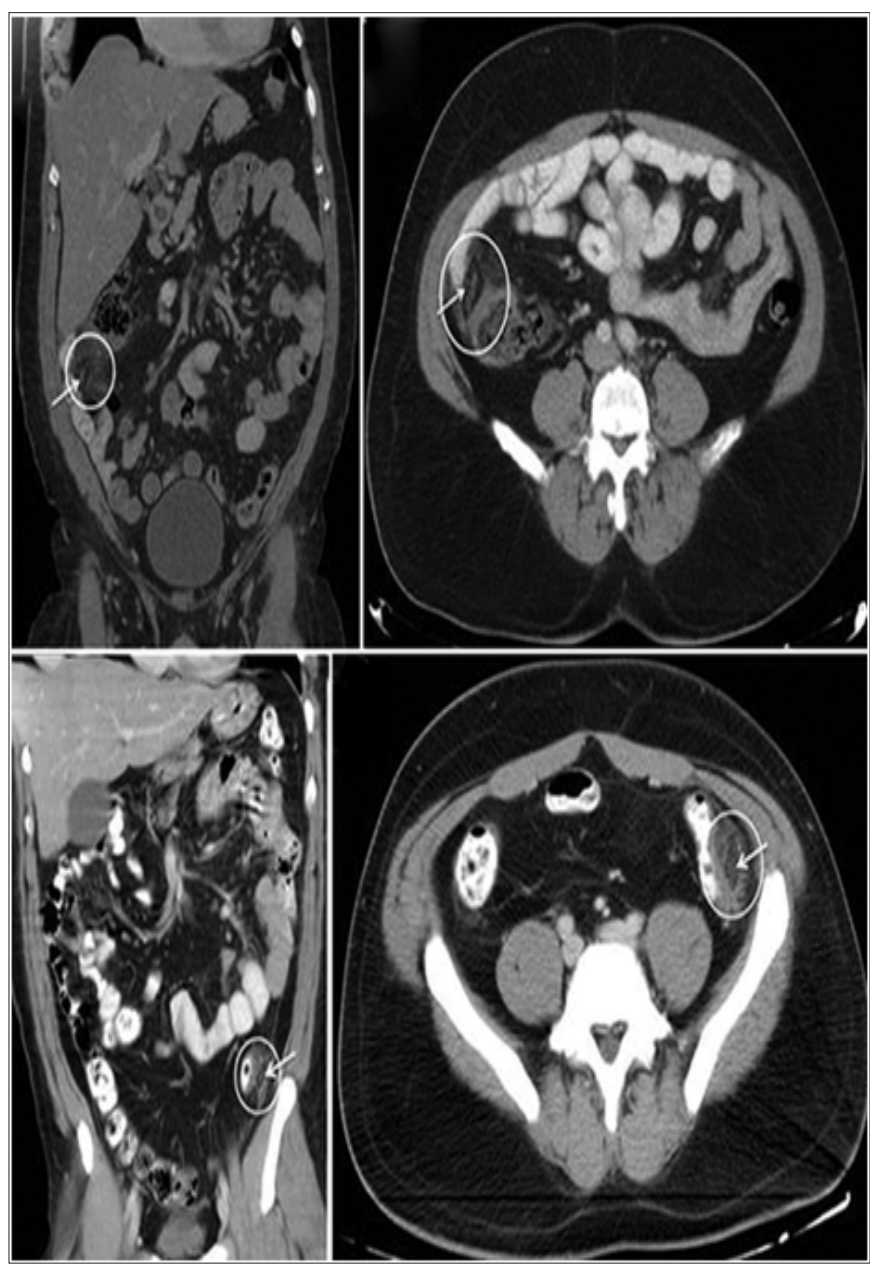

Figure 2: CECT scans of the patient with right lower quadrant abdominal pain (A: coronal reformat, B: axial) and the patient with left lower quadrant abdominal pain (C: coronal reformat, D: axial). CT demonstrated the presence of oval-shaped, fat density paracolonic lesions (white circles) with a high-attenuation central "dot" (white arrow),located at the antimesenteric edge of the ascending colon for the first patient $(A B)$ and the descending colon for the second patient (C, D), respectively. Inflammatory changes of the adjacent pericolonic fat were also detected, more prominent in the second patient. 


\section{Open Access Journal of Surgery}

Primary epiploic appendagitis (PEA) results from spontaneous thrombosis of the veins draining the appendages in absence of any torsion or ischemia [5]. Secondary epiploic appendagitis, on the other hand, develops due to inflammation of adjacent structures like appendicitis, diverticulitis or cholecystitis. PEA often presents with acute abdominal pain and can mimic acute diverticulitis or appendicitis on clinical examination. Its diagnosis is challenging due to the lack of any pathognomonic clinical features. Computed tomography (CT) scan has an important role in the diagnosis of PEA [6]. Timely diagnosis can avoid unnecessary surgical treatment (Figure 1).

\section{Methods}

25 patients diagnosed with primary Epiploic appendagitis were evaluated and analyzed for demographic factors, clinical presentation and diagnostic radiological features. Comparison was also done with data in patients of earlier reported series. All the patients were also followed up for course of disease and treatment. Epiploic appendagitis can occur at any age. In our study the mean age was 44.6 years with a range from 23 74 years. The reported ages range from 12 to 82 years [5]. Men are slightly more affected than women (18 male vs. 7 female in our study) who has also been confirmed by other authors [1]. On clinical examination patients describe a localized, strong, non-migratory, sharp pain which usually started after a specific physical movement of their body like postprandial exercise.

An abdominal tenderness was present in all patients. In our series, patients otherwise felt healthy and rarely described other symptoms. There is a lack of fever, vomiting or leukocytic response. With diverticulitis and appendicitis being the most important causes of lower abdominal pain, they are the most frequent clinical diagnosis before radiologic imaging or diagnostic laparoscopy (Figure 2). The pain usually is on the left or right lower abdominal quadrant [1] (Table 1-4).

Table 1: Showing Clinical features of patients with Appendagitis.

\begin{tabular}{|c|c|}
\hline Clinical Feature & Number (\%) \\
\hline Mean age & $44.6(23-74)$ \\
\hline Sex ratio & $2.5: 1(18: 7)$ \\
\hline Location of pain & $5(19)$ \\
\hline RLQ & $13(54)$ \\
\hline LLQ & $2(8)$ \\
\hline RUQ & $5(27.7)$ \\
\hline Lower Abdomen & $2(13.3)$ \\
\hline Fever $(+)$ & $2(8.1)$ \\
\hline Muscle rigidity $(+)$ & $17(80.6)$ \\
\hline Tenderness $(+)$ & $3(16.6)$ \\
\hline leukocytosis $(+)$ &
\end{tabular}

Table 2: Laboratory Results.

\begin{tabular}{|c|c|c|}
\hline Parameter & Reference Values & Median(min-max) \\
\hline Hemoglobin (g/dl) & $13.5-17.2$ & $13.5(13.1-14.1)$ \\
\hline Hematocrit $(\%)$ & $40-51$ & $40.2(38.5-41.8)$ \\
\hline Leukocytes $/ \mathrm{mm}^{3}$ & $3,500-12,000$ & $6300(5000-9500)$ \\
\hline Neutrophils (\%) & $0-80$ & $61.6(57-65.8)$ \\
\hline Platelets $/ \mathrm{mm}^{3}$ & $140,000-500,000$ & $\begin{array}{c}218,000(175,000- \\
251,000)\end{array}$ \\
\hline Urea (mg/dl) & $10-50$ & $26(17-34)$ \\
\hline Creatinine (mg/dl) & $0.7-1.2$ & $0.8(0.7-0.9)$ \\
\hline $\begin{array}{c}\text { Bilirubin (mg/dl) } \\
\text { C-creative Protein } \\
(\mathrm{mg} / \mathrm{dl})\end{array}$ & $0-1.2$ & $0.5(0.4-0.6)$ \\
\hline
\end{tabular}

\section{Discussion}

Primary epiploic appendagitis is not as rare as is often thought. Its frequency is estimated at $1.3 \%$ in patients presenting with acute pain abdomen [1]. The main presenting symptom is severe, sudden onset, focal abdominal pain with localized tenderness and guarding. However, the symptoms tend to regress within a week. Omental infarct is another entity that has similar clinical and radiological characteristics, but on CT scan, omental infarct often presents as a much larger lesion with no visceral peritoneal thickening [2,3]. Pathognomonic CT scan finding of PEA is a 2-4 $\mathrm{cm}$, oval shaped, fat density lesion, surrounded by inflammatory changes. In contrast to diverticulitis, the colon reveals a uniform diameter with a regular wall and no signs of thickening $[2,3]$. Given its self-limiting course with spontaneously resolution, patients with PEA are conservatively managed [7].

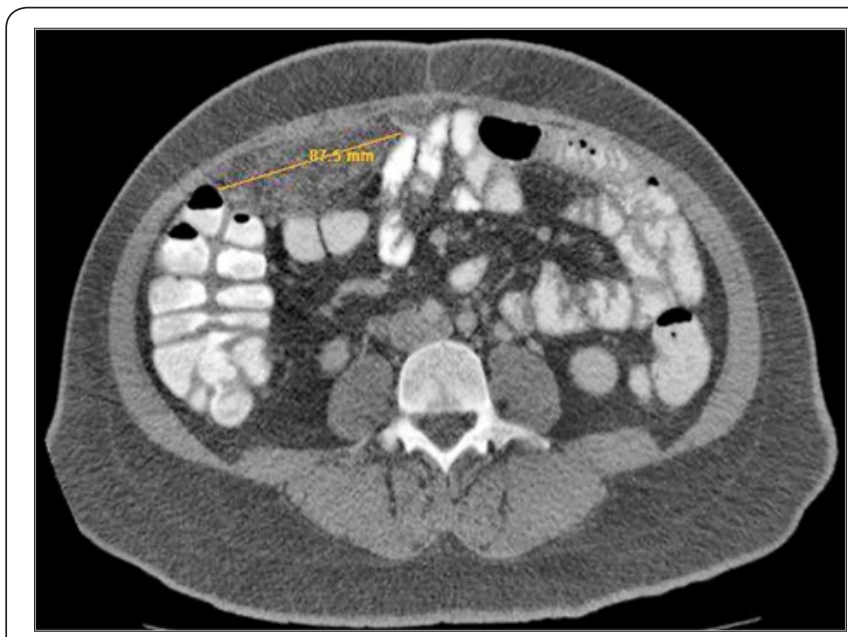

Figure 3 : CECT abdomen showing a large mass in the right mesentery with stranding of fat within and surrounding it.

The most common CT feature in acute epiploic appendagitis is an oval lesion less than $5 \mathrm{~cm}$ in diameter (Typical diameter range $1.5-3.5 \mathrm{~cm}$ ) that has attenuation equivalent to that of fat, abuts the anterior colonic wall and is surrounded by inflammatory 
changes [1]. Thickening of the visceral peritoneum secondary to the spread of inflammation also may be observed. The wall of colon may be thickened but is most often normal in thickness [1]. Although the presence of a central area of high attenuation due to venous thrombosis is useful for diagnosis, the absence of this feature does not preclude a diagnosis of acute epiploic appendagitis (Figure 3).

Almost all of the above mentioned findings were present in our cases. Sometimes the central high attenuation dot/area calcifies. This small calcified body may become detached and appears as a "Peritoneal loose body" in the abdominal cavity. It may reattach itself to a surface such as the lower aspect of spleen in which case it can be called a "Parasitized appendix epiploic". In the past, diagnosis of EA was often the result of an unexpected finding at exploratory laparotomy. Today, however, a variety of ultrasound (US) and CT findings has been described which in some cases aids the surgeon to make the right diagnosis preoperatively.

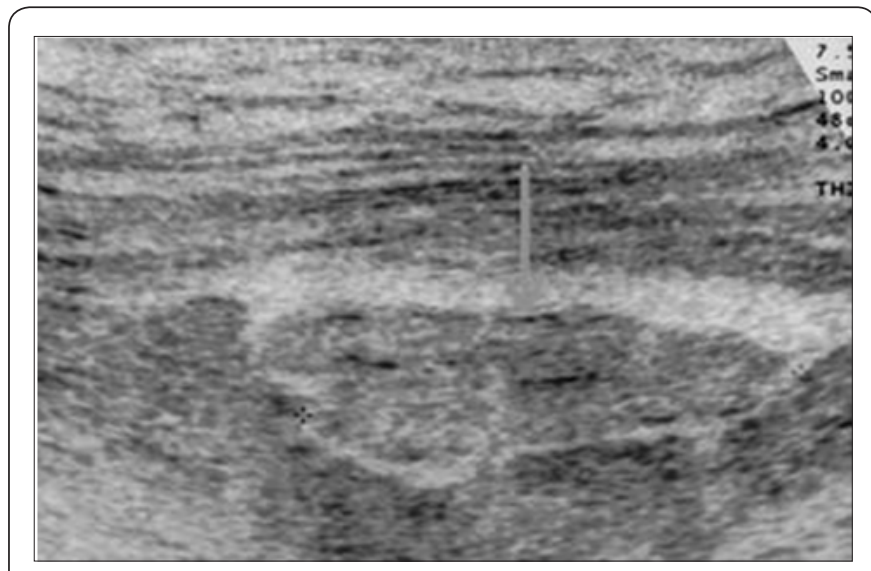

Figure 4 : Ultrasonography showing oval hyperechoic lesion in iliac fossa with a thin rim of hypoechoic fluid around it.

US sometimes show an oval, non-compressible hyperechoic mass with a subtle hypoechoic rim directly under the site of maximum tenderness. There is no central blood flow depicted on color Doppler US imaging. The latter described picture was observed in 8 of 25 patients (32\%). Ultrasound in combination with CT scan made the correct preoperative diagnosis possible in 14 of 25 patients. Normal epiploic appendages are not seen on CT scan (Figure 4). They typically have fat attenuation and cannot be distinguished from other adipose structures like retroperitoneal fat unless they are surrounded by intraperitoneal fluid or inflammation. With the introduction of cross-sectional imaging and the increasing use of abdominal CT scan for primary evaluation of lower abdominal pain the recognition of EA has increased. In 1986 Danielson et al. [2,3] were the first that reported an EA diagnosed by CT scan.

Meanwhile this entity has become more common and numerous reports describing CT scan features have been published. Pathognomonic CT scan findings are a $2-4 \mathrm{~cm}$, oval shaped, fat density lesion, surrounded by inflammatory changes. One can distinguish a central focal area of hyperattenuation with surrounding inflammation. Thickening of the parietal peritoneum wall can be sometimes observed. In contrast to diverticulitis the diameter of the colonic wall is mostly regular without signs of thickening. The diagnosis of epiploic appendagitis has become easier with the development of high resolution computed tomography (CT), and this has been recently supported in the literature.

On CT studies, round or ovoid lesions of fatty density measuring $1.5-3.5 \mathrm{~cm}$ in diameter with a hyper attenuating rim and surrounding ill-defined fat stranding are characteristically identified in the mesenteric fat adjacent to the colon. The mesenteric fat in these lesions is typically higher than normalappearing mesenteric fat elsewhere in the abdomen $[2,3]$. The hyper attenuating ring is considered to be a characteristic sign [5]. Fat stranding is more pronounced than wall thickening because the Para-colonic inflammatory changes are disproportionately more severe than the mild local reactive thickening of the adjacent colonic wall $[2,3]$.

In patients with acute abdominal pain this suggests a relatively narrow differential diagnosis: diverticulitis, omental infarction, appendicitis and less commonly mesenteric panniculitis $[4,5]$. Many studies have reported that CT scan is useful to rule out the need for surgical treatment [7]. In our patient, antibacterial therapy and fasting were initiated on the first day of hospitalization, but his symptoms worsened on the third day of hospitalization. Secondary epiploic appendagitis and abscess formation were ruled out through subsequent CT scan. Mild diverticulitis was considered difficult to distinguish from PEA. Computed tomography scan showed that the lesion was a solid, lobular-like soft-tissue density mass, and it was not adjacent to the colon wall. On the basis of these characteristic findings, we diagnosed the lesion as PEA, not diverticulitis. In the case of diverticulitis, the interior of the lesion is not solid, and it has a three-layer structure.

That is, there is a hyper-attenuated mucosal layer from the inside, thickened sub-mucosa with a low density, and a large amount of serosa. Oral administration of non-steroidal antiinflammatory drugs (NSAIDs) was initiated, and his symptoms subsequently improved. This indicates that NSAIDs may be more effective for the treatment of PEA than antibiotics. In conclusion, in a patient presenting with sharp, localized, acute abdominal pain unassociated with nausea, vomiting, fever or laboratory values typical of acute abdomen, a differential diagnosis of PEA should be entertained, and a CT scan abdomen should be performed to clinch the diagnosis.

\section{Conclusion}

Epiploic appendagitis is a surgical diagnosis with clinical features that may guide the surgeon to the right pre-operative diagnosis. In patients with localized, sharp, acute abdominal 
pain which is not associated with other symptoms like nausea, vomiting, fever or typical abdominal laboratory values, the diagnosis of EA should be considered as a differential diagnosis to sigmoid diverticulitis and appendicitis. Although infrequent until now, with the increase of primary abdominal CT scans and ultrasound, which have become standard diagnostic imaging tools, EA will be diagnosed more frequently in the future. This study describes the clinical features of EA as a possible guide to the surgeon for the correct diagnosis of this rare disease. Epiploic appendagitis was more frequent in males. Abdominal pain was located in the lower quadrant, more predominant in the left than right. Blood tests were normal except for increased levels of C-reactive protein. Diagnosis was made mostly preoperatively due to imaging tests, avoiding unnecessary surgical intervention.

\section{References}

1. de Brito P, Gomez MA, Besson M, Scotto B, Huten N, et al. (2008) Frequency and epidemiology of primary epiploic appendagitis on CT in adults with abdominal pain. J Radiol 89(2): 235-243.
2. Rao PM, Wittenberg J, Lawrason JN (1997) Primary epiploic appendagitis: evolutionary changes in CT appearance. Radiology 204: 713-717.

3. Pereira JM, Sirlin CB, Pinto PS, Jeffrey RB, Stella DL, et al. (2004) Disproportionate fat stranding: a helpful CT sign in patients with acute abdominal pain. Radio Graphics 24(3): 703-715.

4. Desai HP, Tripodi J, Gold BM, Burakoff R (1993) Infarction of an epiploic appendage. Review of the literature. J Clin Gastroenterol 16(4): 323325.

5. Rioux M, Langis P (1994) Primary epiploic appendagitis: clinical, US, and CT findings in 14 cases (1994) Radiology 191(2): 523-526.

6. Macari M, Laks S, Hajdu C, Babb J (2008) Caecal epiploic appendagitis: an unlikely occurrence. Clin Radiol 63(8): 895-900.

7. Ghosh BC, Shatzkes J, Webb H (2003) Primary epiploic appendagitis: diagnosis, management, and natural course of the disease. Mil Med 168(4): 346-347. 